\title{
Real de Minas de San Gregorio de Mazapil, frontera del Reino de la Nueva Galicia
}

Juana Elizabeth Salas Hernández ${ }^{1}$

\section{RESUMEN}

La conformación territorial de la Monarquía Hispánica estuvo constituida por un proceso de territorialidad y desterritorialidad, procesos que estuvieron marcados por la conformación de fronteras administrativas, sociales, imaginarias, geográficas y ambientales, mutables en el espacio y en el tiempo. El Reino de Nueva Galicia en su conformación territorial durante los siglos XVI-XVII estuvo en una expansión y conquista constantes y por lo tanto la Tierra Adentro se fue modificando, y con ella la frontera. San Gregorio de Mazapil fue un Real de Minas, que desde su fundación en 1568, fungió como frontera administrativa entre el Reino de la Nueva Galicia y el de la Nueva Vizcaya, pero también fue frontera social, de guerra, geográfica y ambiental. El artículo analiza ese Real de Minas como estudio de caso, y reflexiona esas categorías, concentrándolas en la frontera social, de guerra, geográfica y ambiental, para comprender el proceso de poblamiento y la transformación del paisaje durante los siglos XVI-XVII.

Palabras clave: territorio; frontera; transformación del paisaje; Nueva Galicia. 
$\mathrm{L}$

a conformación territorial de la Nueva Galicia ha sido muy discutida en la historiografía neogallega. En la mayoría de los trabajos historiográficos se encuentra la preocupación explícita o implícita por explicar la delimitación geográfica del reino, lo cual es de suma importancia para comprender su proceso histórico.

En la historiografía de la Nueva Galicia ha existido la preocupación de analizar y entender cómo fue la conformación territorial de la Nueva Galicia (Véase mapa 1). Siguiendo esta línea, el objetivo de este artículo es analizar el proceso de conformación y expansión territorial de la Nueva Galicia ${ }^{2}$ teniendo como estudio de caso al Real de Minas de San Gregorio de Mazapil, ${ }^{3}$ el cual nos permitirá reflexionar sobre el concepto de frontera desde diversas aristas, todo con la intención de comprender las transformaciones medio ambientales que desembocaron en la transformación del paisaje durante los siglos XVI y XVII (Véase mapa 2).

Mapa 1. Audiencia de la Nueva Galicia, siglo XVI
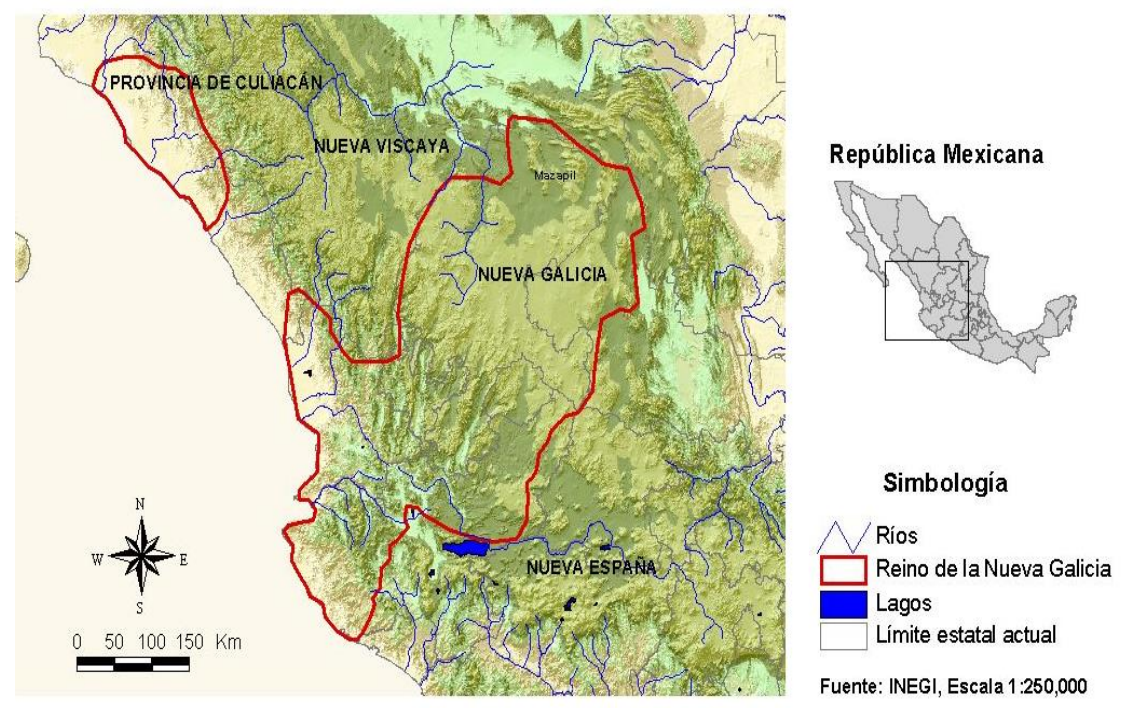

Fuente: Los límites de la Audiencia de la Nueva Galicia en el siglo XVI según los límites ordenados por el Virrey Antonio de Mendoza, y los señalados en la obra de Juan López de Velasco. Elaboración propia, con base en el documento AGI, AGI, PATRONATO182, R.45.

\footnotetext{
2 El Reino de la Nueva Galicia estuvo conformado por los actuales estados de Nayarit, Jalisco, Aguascalientes, Zacatecas, partes de Guanajuato y San Luis Potosí en México.

${ }^{3}$ Actualmente Mazapil es municipio del estado de Zacatecas, México.
} 
Mapa 2. Reales de minas de la Nueva Galicia

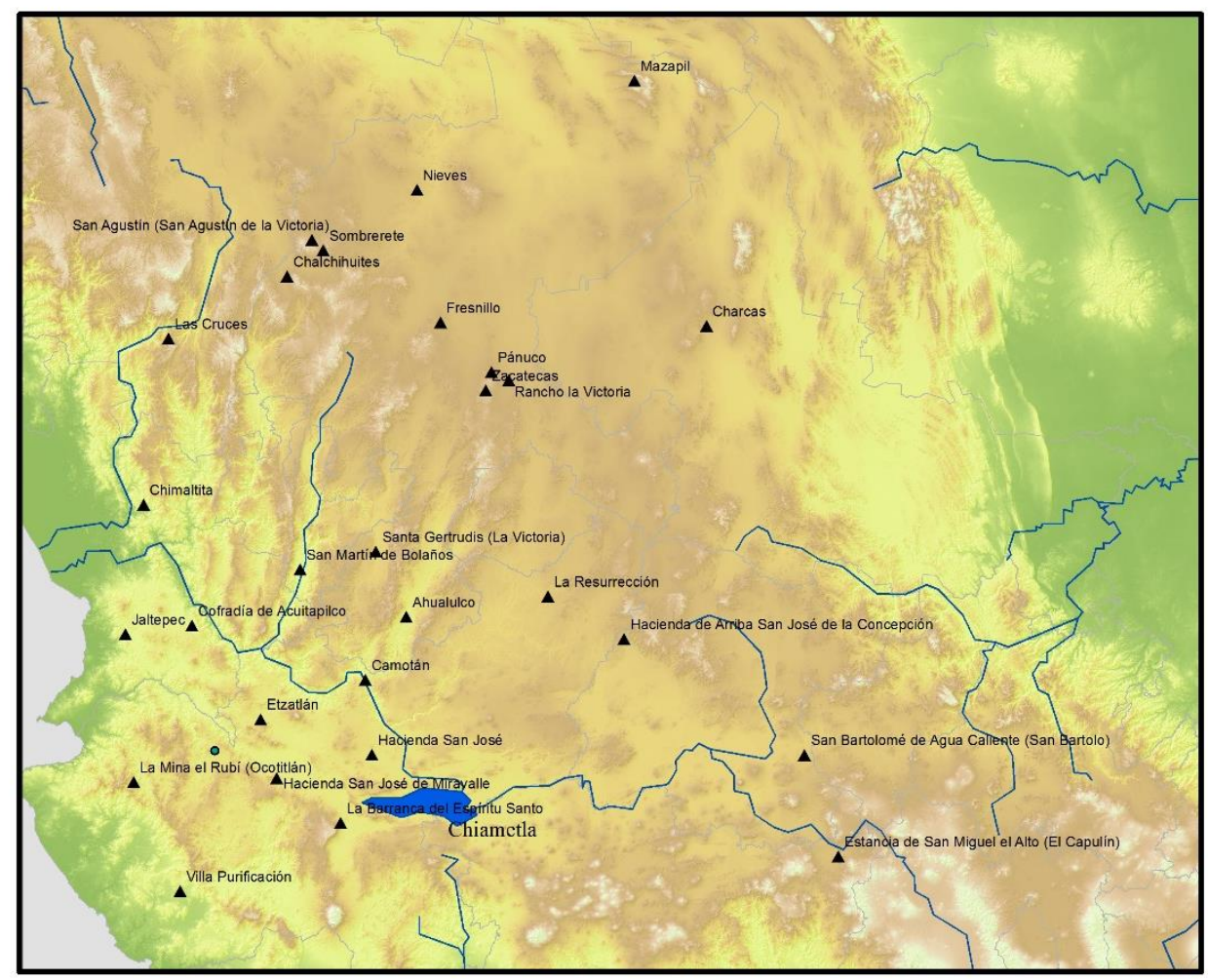

Fuente: Elaboración propia

En la historiografía se ha usado el concepto de territorio para referir a la actuación de los humanos en un espacio específico. Lo defino como, un espacio delimitado por las acciones humanas, no sólo en el ámbito administrativo, sino que se entiende al territorio como el espacio habitado; "desde la perspectiva social, el territorio puede entenderse como un conjunto que interactúa; un espacio, que al mismo tiempo, rivaliza con otros territorios: las relaciones territoriales conforman entonces un espacio de interacciones compuesto por fronteras móviles que lo hacen dinámico y flexible". 4

El concepto de territorio se vincula con el de territorialidad, la cual es "el conjunto de relaciones que una población mantiene en un territorio percibido como 
suyo y con las dinámicas provenientes del exterior". ${ }^{5}$ Partimos de la premisa de que los estudios del territorio deben basarse en la interdisciplinariedad, esto permite que el concepto de territorio transite de los estudios geográficos a otras disciplinas y con ello lograr un diálogo que permita comprender al territorio como un laboratorio social. Para su comprensión se han utilizado diversas escalas de análisis, marcos teóricos y metodológicos. ${ }^{6}$

La historia ambiental ha permitido analizar la interacción entre grupos sociales y el medio ambiente; es así que combinado este enfoque con la dimensión temporal, podemos comprender procesos de territorialidad y desterritorialidad; y con ello la mutación de las fronteras.

La conformación del territorio es dimensionada en el tiempo, por lo cual se utilizará el concepto de cronotopología, propuesto por Juan Pedro Viqueira; ${ }^{7}$ este concepto ayuda a estudiar sistemáticamente el espacio en el tiempo. Partiendo de la idea de que el territorio de la Nueva Galicia fue una construcción, que estuvo marcada por continuidades y rupturas. Una de ella fue, el conflicto entre los indígenas, habitantes originarios, en el sentido que estuvieron ocupando el territorio antes que los españoles. La historia oficial ha enseñado que los ganadores fueron estos últimos. El proceso histórico fue más complejo, que el sólo hacer la división, entre vencedores y vencidos. En la construcción del territorio neogallego queda claro que los indígenas no tuvieron un papel pasivo.

Otra ruptura es la construcción española del territorio. Pareciera que los españoles sólo vertieron el esquema que ya traían, marcado por delimitaciones administrativas y personales. Creemos que fue más una adecuación a las particularidades geográficas y ambientales del espacio que se iban encontrando con sus concepciones territoriales, por lo que coincido con la idea de José Francisco Román de que, "el territorio era un elemento en continua transformación al imprimir diversas

\footnotetext{
${ }^{5}$ Arturo Taracena Arríola, "Región e historia", Desacatos. Revista de antropología social, revista semestral, (primavera de 1999), 29.

6 Véase, Raúl Fonseca Hernández, Wilson Picado Umaña, Abelardo Morales Gamboa y Maximiliano López López, eds., Cruce de caminos. Lecturas disciplinarias del territorio (San José de Costa Rica: FLACSO Costa Rica/Universidad Nacional de Costa Rica: 2020).

7 Miguel J. Hernández Madrid y José Lameiras Olvera (eds.), Las ciencias sociales y humanas en México. Síntesis y perspectivas del fin de siglo (México: El Colegio de México, 2000).
} 
dinámicas a la acción humana bien que se contemplara a través de la mirada de los europeos, bien de los indígenas". ${ }^{8}$

Con estas precisiones analizamos a Mazapil como un territorio de frontera, con el objetivo de explicar la territorialidad en un contexto de conflicto, que derivó en la internalización de nuevas formas de apropiación del espacio, "permitiendo de emergencia de identidades construidas por las mismas sociedades indígenas como respuesta de la transformación externa de sus espacios de vida, principalmente, a la transformación de las relaciones sociales como resultado de los procesos de conquista y colonización". ${ }^{9}$

En el proceso de exploraciones y conformación del territorio se fueron descubriendo lugares que fueron importantes para el avance fundacional y poblacional, en la mayoría de las ocasiones se desarrolló en conflictos bélicos, sociales y de contenido ambiental. ${ }^{10}$ En ese contexto fue en el que se descubrió en 1568, Mazapil, que se convertiría en el Real de Minas de San Gregorio de Mazapil.

El valle de Mazapil, tenía el nombre de Mazatlpilli, nombre de origen náhuatl; aún la búsqueda documental e historiográfica no ha podido aclarar el momento en el se comenzó a utilizar esta denominación. Fue con el descubrimiento de las minas por parte de los españoles que el valle se nombró, San Gregorio, patrono de los mineros. Al adquirir la categoría de Real de Minas, fue como el nombre sería "Real de Minas de San Gregorio de Mazapil". El inicio del poblamiento español ha sido referenciado por la llegada del capitán Enríquez, 16 soldados y el escribano Pedro Valverde. ${ }^{11}$

Para 1569 en la jurisdicción de Mazapil se contaba con 150 vecinos y para 1573 solo había 30, la reducción quizá se debió a un desabasto de alimentos, es aún tema pendiente en la historiografía. Teniendo en cuenta que el término de vecino indicaba al jefe de una familia, se han podido rastrear algunos de los nombres de los que se consideran los primeros pobladores españoles del Real de Minas; sin embargo es

\footnotetext{
8 José Francisco Román Gutiérrez, Sociedad y evangelización en la Nueva Galicia durante el siglo XVI (Guadalajara: Instituto Nacional de Antropología e Historia/El Colegio de Jalisco/Universidad Autónoma de Zacatecas, 1993), 123. 1َ'sêpi

9 Sheridan Prieto, "Reflexiones", 77 .

10 Véase: Mauricio Folchi D, "Conflictos de contenido ambiental y ecologismo de los pobres: no siempre pobres, ni siempre ecologistas" Ecología Política (22 2001), 79-101.

11 Pedro Ascacio Ortiz, "Mazapil", Los municipios de Zacatecas. Memoria y Patrimonio (Zacatecas, México: Crónica del estado de Zacatecas, 2019), 232.
} 
importante tener en cuenta el crisol étnico que para finales del siglo XVI existía en este lugar (Véase mapa 3). ${ }^{12}$

Mapa 3. Poblamiento de Mazapil
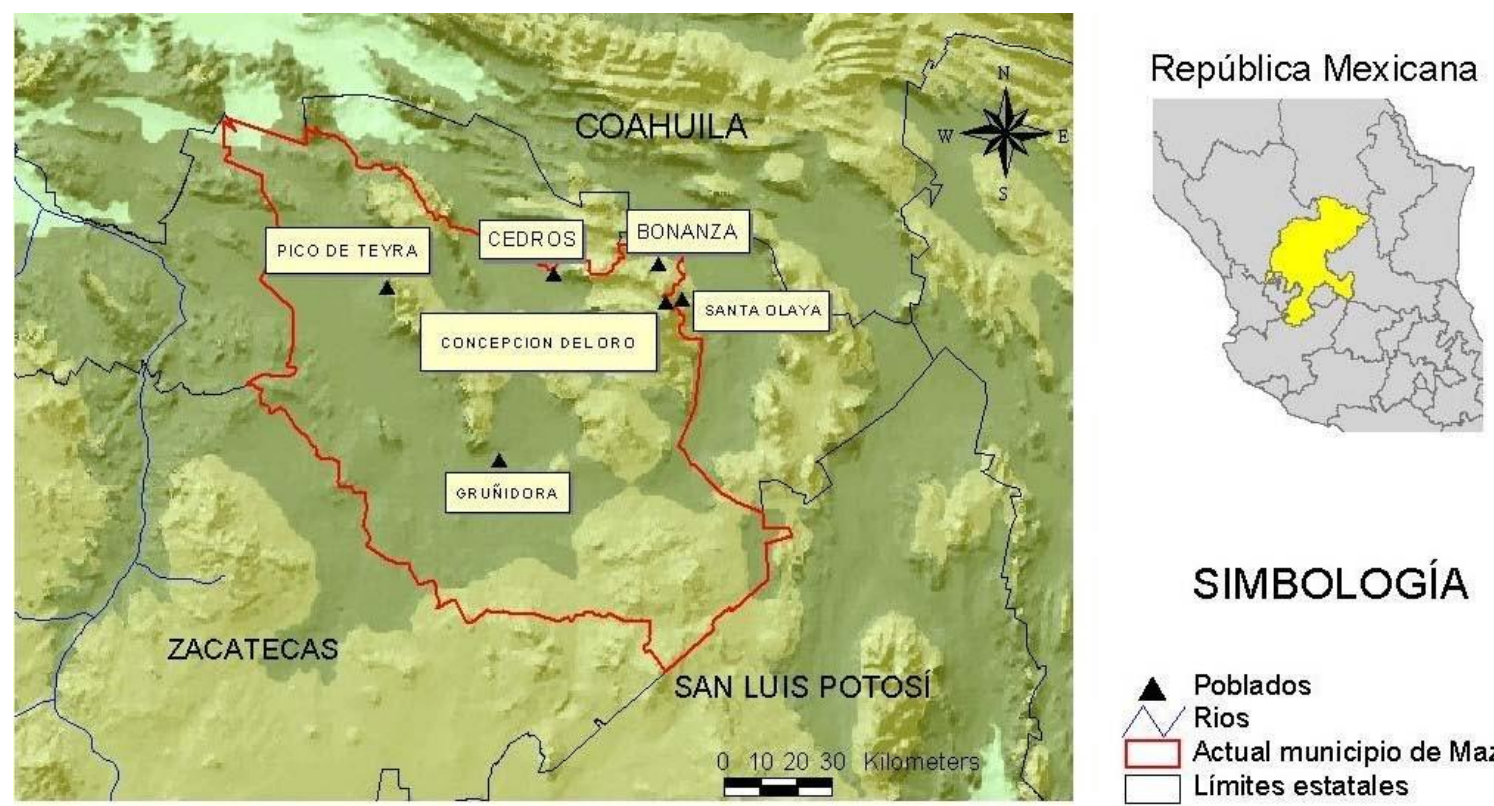

\section{SIMBOLOGÍA}

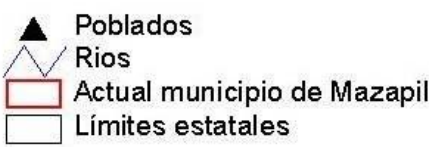

Fuente: Elaboración propia

El artículo está divido en tres secciones, en la primera se exponen las circunstancias históricas de la fundación y poblamiento de Mazapil; en la segunda se describe la frontera geográfica y ambiental, y en la tercera se describe la frontera de guerra en el contexto de la guerra chichimeca.

\section{SAN GREGORIO EN LA FRONTERA}

Durante el siglo XVI, el territorio de la Nueva Galicia estuvo en construcción, dependiente de las exploraciones y la consecuente formación de poblados,

\footnotetext{
12: Francisco Valverde (alcalde mayor), Francisco Cano (Teniente de alcalde mayor), Pedro Valverde (escribano real), Diego de Zuolaga, Pedro Arriola, Melchor de Calahorras, Pedro Crespo, Miguel Esteban, Pedro Esteban, García Dávila, Alonso González, Pedro Jiménez, Francisco Leyva, Pedro López, Juan López Godoy, Pedro Lorenzo, Diego Martínez, Juan de Minchaca, Álvaro Méndez, Alonso Pérez Suárez, Gaspar de Rivera, Francisco Rodríguez, Francisco de Torquemada, Miguel Torres, Alonso Zapata, Martín de López (vicario), Andrés Ruiz de Alarcón (vicario). AGI, Patronato 22, R. 3, 39- 40; AGI, Indiferente General 1092; AGI, Contratación 210, N.3.
} 
independientemente de la categoría administrativa que se les otorgó desde la administración hispánica: pueblo de indios, ciudad, villa o real de minas. Este proceso territorial que conformó el rostro hispanizado del espacio; que al ser "parte de la sociedad colonial quedaba inscrito en un proyecto de organización y creación de instituciones, costumbres, formas de vida, normas, así como conductas prácticas de los individuos que habitaron esa región". ${ }^{13}$

Se puede decir que las relaciones de territorialidad en la construcción de territorio fueron de frontera. "En otras palabras desde su origen, el asentamiento colonial de Nueva Galicia fue territorio de frontera en su dimensión social, no sólo por una naturaleza difícil de transformar, o por la respuesta violenta de los chichimecas cuando sus territorios de supervivencia fueron ocupados". ${ }^{14}$

La frontera fue movible debido a la expansión española, por el afán de ir encontrando lugares, así que cada nuevo descubrimiento significaba una nueva frontera (véase mapa 3).

En la segunda década del siglo XX, Frederick Turner, introdujo el concepto de frontera, a los estudios históricos, como explicación para la civilización americana, él creía que "la existencia de una frontera explicaba gran parte de las diferencias existentes entre Europa y el Nuevo Mundo"; 15 así que ésta era la división entre la civilización y la barbarie. La idea de Turner resultó imprecisa, ya que a veces se refería, a un proceso y otras, a un lugar. Hebert Eugene Bolton, fundador de la escuela de la frontera, rechazó que fuera sólo una división entre la civilización y la barbarie, se preocupó por elaborar una definición que incluyera a las personas, así que concluyó que en ella había una interacción entre dos culturas diferentes.

\footnotetext{
${ }^{13}$ Gil González Dávila, editor, Guerra de los chichimecas (México: Gobierno del Estado de Jalisco/Universidad de Guadalajara/Instituto Jalisciense de Antropología e Historia 1994), 426.

14 González Dávila, Guerra de los chichimecas, 422.

15 Francisco de Solano y Salvador Bernabéu coords., Estudios (nuevos y viejos sobre la frontera) (Madrid: Consejo superior de investigaciones científicas-centro de estudios históricos, departamento de América 1991), 61.
} 
Mapa 4. Jurisdicción de Mazapil, siglo XVII

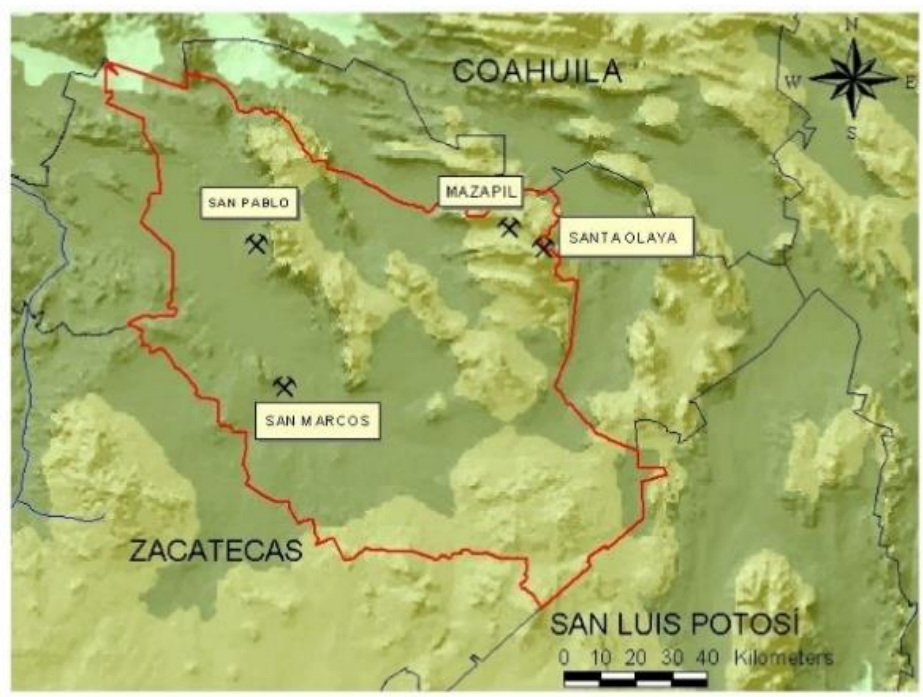

República Mexicana

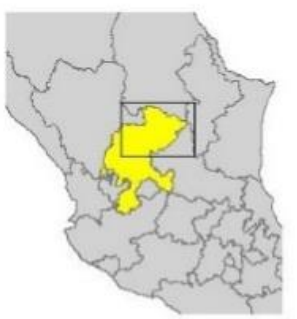

SIMBOLOGÍA

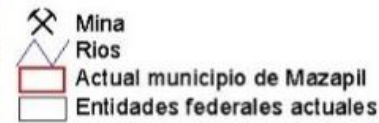

Fuente: Elaboración propia con base en Minas en la jurisdicción de Mazapil durante los siglos XVI y XVII. AMM, FONDO COLONIAL, CAJA 1, EXPEDIENTE1, Testamento del Capitán Francisco de Elizondo (3 junio 1653-1 junio 1656); Garza Martínez y Pérez Zevallos, El Real..., pp. 40-45.

Georges Baudot propuso que la guerra chichimeca, se desarrolló en seis distintas fronteras: agrícola, ganadera, minera, militar e imaginada. La primera, la identificó en espacios rurales, donde se practicaba el cultivo comercial de la tierra "con una producción destinada a mercados de mayor o menor amplitud en que se negocian las cosechas y que en cierto modo marcan geográficamente ciudades fundamentadas en la explotación agrícola". ${ }^{16}$

La minera, marcada por las fundaciones provocadas por las explotaciones mineras, es decir los reales de minas; podía confluir con la agrícola y la ganadera; y a veces con la militar, se ubicaba en las áreas marginales del virreinato, y era creada antes que la religiosa. Era un "asentamiento de frailes pioneros para las labores de evangelización, que viven en contacto con los grupos amerindios del entorno geográfico que sueñan con integrar (...) desfronterizar y que llevan por marca diversas características bien peculiares" ${ }^{17}$ La propuesta de Baudot ayuda a explicar la manera

\footnotetext{
${ }^{16}$ A. F Buenrostro Ceballos, ed., Memoria del I Congreso Internacional sobre Fronteras en Iberoamérica: Ayer y hoy (Tijuana: Universidad Autónoma de Baja California, Tomo 1), 208.

17 Buenrostro Ceballos, Memoria, 209.
} 
hispana de espacializar el nuevo territorio; su tesis más importante, fue el último tipo de frontera, la imaginada, sutil y quimérica, "son las fronteras de aquellos espacios aún desconocidos, por explorar y por entender, que deslindan y marcan los límites de reinos imaginarios, de ciudades fantásticas, en todo el proceso complejo del reconocimiento de América y de su conquista y control". ${ }^{18}$ La hipótesis del autor es que esta fue la que dio valor a los europeos de introducirse en tierras desconocidas y peligrosas.

La categoría de frontera imaginada es muy interesante, su carácter simbólico, la hace difícil de rastrear, sin embargo ayuda para explicar como los españoles importaron su bagaje cultural, para explicar lo nuevo que veían sus ojos. Se le puede entender como, "un espacio donde se produce la transición, la interacción y los conflictos entre grupos humanos que se reconocen diferentes". ${ }^{19}$ El propósito de utilizar el concepto de esta manera es entender cómo se trasformaron los grupos sociales involucrados en el poblamiento de el Real de Minas de San Gregorio de Mazapil, y cómo transformaron el paisaje.

Siguiendo las propuestas teóricas mencionadas, podemos decir que la frontera neogallega, se divide en tres categorías de fronteras: de guerra, geográfica y ambiental; éstas se analizan para explicar a Mazapil como un territorio de frontera. La dinámica de poblamiento en este lugar, y las relaciones de frontera con el espacio, fueron particulares, marcadas por permanencias y rupturas, como lo señala el concepto de cronotopología.

En los siglos XVI y XVII, el concepto de frontera fue utilizado en cuatro sentidos: primero, eran los lugares que aún no habían sido pacificados, "Los españoles se referían con el término frontera a todos los enclaves de la colonización situados en regiones donde los indios no estaban todavía pacificados del todo". ${ }^{20}$ En segundo lugar como, un límite territorial; el tercer sentido, una frontera social, recordando que la territorialidad en la Nueva Galicia estuvo marcada por la tensión entre dos grupos, los españoles y los indígenas. Los primeros llegaron a ocupar un territorio que ya pertenecía a los

\footnotetext{
${ }^{18}$ Buenrostro Ceballos, Memoria, 211.

19 José Alfredo Rangel Silva, "Capitanes a guerra, linajes de frontera. Estrategias de dominación entre las élites familiares en el oriente de San Luis, 1617-1823", México, (Tesis doctoral, El colegio de México, 2006), 12.

20 Chantal Cramaussel, Poblar la frontera. La provincia de Santa Bárbara en Nueva Vizcaya durante los siglos XVI y XVII, (México, El Colegio de Michoacán: 2006), 21
} 
segundos, por lo tanto hubo una reacción de estos, que se ha denominado guerra, provocada por la "presencia culturalmente distintas que en el ataque buscan resolver o preservar sus entornos de supervivencia casi es tan antigua como la fundación misma de las villas de españoles". ${ }^{21}$ El cuarto sentido, tiene que ver con la movilidad de la frontera debido a los nuevos descubrimientos, y una consecuente pacificación necesaria en una zona de frontera. Es así que la presencia de las órdenes mendicantes fue prioritaria,

el recorrido que hacían los franciscanos en la frontera de guerra modificó la relación entre el mundo indígena y el español. Conforme se iban descubriendo nuevas zonas, o pacificando relativamente algunos grupos chichimecas, no solo se producía un desplazamiento en los territorios que era posible ocupar-propiciando el acceso a nuevos descubrimientos-sino también se iban identificando mejor los indígenas y sus diferentes asentamientos, aunque poco caso se hizo de las costumbres, lenguas y ritos. $^{22}$

\section{Frontera GEOGRÁfica y AMBIENTAL}

La frontera en el territorio de la Nueva Galicia fue movible, durante el siglo XVI y siglos posteriores; así que cada nuevo poblamiento, significaba una nueva frontera, "el último asiento, casi siempre, servía de base para realizar otras entradas en las tierras desconocidas". ${ }^{23}$

Por lo tanto los límites no eran definidos. La fundación de Mazapil, significó el reforzamiento del acceso del norte, y los límites con el reino de la Nueva Vizcaya. Alonso de la Mota y Escobar en su Descripción de los reinos de la Nueva Galicia, Nueva Vizcaya y Nuevo León, escrita entre 1604 y 1607, señaló: "Este real de minas del Mazapil es la raya y el último término de este Reino de la Nueva Galicia, por estar parte más

\footnotetext{
21 Román Gutiérrez, Sociedad y evangelización, 360.

22 Román Gutiérrez, Sociedad y evangelización, 365.

${ }^{23}$ Román Gutiérrez, Sociedad y evangelización, 414. Para el estudio de las relaciones entre franciscanos y guachichiles véase: Martínez Sánchez Lucas, Guachichiles y franciscanos. En el libro más antiguo del convento de Charcas 1568-1663, (México: Gobierno del estado de Coahuila de Zaragoza, Secretaría de Cultura, Consejo Editorial del Estado, 2019).
} 
arrumbada al norte". ${ }^{24}$ Esta cita nos permite explorar a Mazapil como frontera geográfica.

Mazapil tuvo una posición estratégica al estar situado en el semidesierto, "llegando a concentrar algunos de estos indígenas como trabajadores en el real de minas, a la vez que proporcionaba suministros y descanso a quienes viajaban hacia reales más septentrionales o que se encontraban próximos al Nuevo Reino de León", ${ }^{25}$ y con el Reino de la Nueva Vizcaya. Las primeras descripciones de este real de minas, señalan que era un lugar inhóspito, por sus características ambientales: "es tierra mas fría y toda despoblada por que los naturales no tienen asiento que es gente feroz y salteadores todos ellos y son en cantidad de mas de dos mil con los que se han hallado en una legua que adelante otras veinte leguas habrá treinta españoles poblados en Mazapil y cada día tienen conflictos con los indios y se matan unos a otros tienen poca esperanza de que esta población permanezca así con los naturales no se aquietan". ${ }^{26}$

En ese medio ambiente con características desérticas, era difícil que el poblamiento español durara, según los pronósticos que hicieron algunos observadores, como los obispos, los oidores y los capitanes de guerra. En el libro de cuentas de 1608, se describe como: "hay cincuenta leguas todas despobladas, y tierra estéril de pastos y aguajes porque es salitral de manera que solo animales silvestres y yeguas cimarronas habitan en ella y las aguas son salubres, carecen de ella los caminantes porque caminan todo el día no tienen más que un aguaje en cada uno, por haber de un aguaje a otro ocho y doce leguas. Y su temple frío y muy airoso y el polvo muy perjudicial para la salud".27

Los españoles se enfrentaron al conflicto de habituarse a un lugar en el que para ellos resultaba difícil vivir, no sólo por los enfrentamientos con los guachichiles, ${ }^{28}$ sino también por las características ambientales. Cuestión que favoreció a los últimos que

\footnotetext{
${ }^{24}$ Alonso de la Mota y Escobar, Descripción de la Nueva Galicia, Nueva Vizcaya y Nuevo León, (México: Ed. Pedro Robredo, 1940), 161.

${ }^{25}$ Román Gutiérrez, Sociedad y evangelización, 414.

${ }^{26} \mathrm{AGI}$, GUADALAJARA 55, 2.

${ }^{27}$ AGI, CONTADURÍA 874, 1608-1672, 373v.

${ }^{28}$ Chichimeca es un nombre genérico, 'de sentido descriptivo que utilizaban los nahuas y que después fue adoptado por los españoles, para referirse a los habitantes del norte" (Lazos Ruiz Adi Estela y Claudio Garibay Orozco, "legados chichimecas en el paisaje", Salas Hernández Juana Elizabeth y Margil de Jesús Canizales Romo, Historia ambiental en el Norte de México, (México, Universidad Autónoma de Zacatecas, El Colegio de San Luis A.C., Red Columnaria, 2020), 71. Los estudios historiográficos y arqueológicos han definido a los guachichiles como integrantes de las culturas del desierto. En Mazapil era uno de los grupos étnicos que habitaban el territorio antes de la llegada de los españoles.
} 
estaban en su territorio natural, "Esta población y real de minas está en frontera de los guachichiles y es la tierra más expugnable para ello de toda la gala [sic] y desde donde hicieron más daño porque son serranías muy ásperas y frías sin agua y como ellos la tiene en unos magueyes pequeños de que hay abundancia". ${ }^{29}$

La convivencia en esa frontera geográfica y ambiental. significó un proceso lleno de complicaciones y adecuaciones para los grupos étnicos que lo habitaron. Los guachichiles sufrieron también una adaptación a la nueva territorialidad introducida e impuesta por los españoles. Ese proceso fue dinámico, y produjo una relación reciproca entre los dos grupos sociales señalados, que convivieron con características ambientales en las que se basaron la formación de la ontología histórica del Real y Minas de San Gregorio de Mazapil.

Como han demostrado Francesco Panico y Claudio Garibay, la transformación en el paisaje del Valle de Mazapil durante la época virreinal estuvo trazado por el carácter agrominero que adquirió a partir del poblamiento español en 1568. La posición territorial de Mazapil y su cercanía y comunicación con lugares tan importantes como Zacatecas, le otorgaron una ubicación estratégica. ${ }^{30}$

La dinámica poblacional también provocó la fundación de otros pueblos, haciendas de beneficio, estancias de ganado. Entre las primeras, ya para finales del siglo XVI, se encontraban: San Juan de Cedros y Bonanza; en cuanto a las estancias de ganado y carboneras estaban, Coapas, San Tiburcio y Gruñidora. Las minas más importantes dentro de la jurisdicción de Mazapil fueron: "Santa Olaya y Santa Isabel a unos 12 kilómetros del sureste de Mazapil; Albarradón que era de las más cercanas, a unos 8 kilómetros al este; el Peñasco a 14 kilómetros al oeste; el Pedregoso y Zuolaga entre unos 45 y 50 kilómetros al noreste y al norte, respetivamente". ${ }^{31}$

\footnotetext{
${ }^{29} \mathrm{AGI}$, CONTADURÍA 874, 1608-1672, 374

30 Panico Francesco y Claudio Garibay, Mazapil, Zacatecas, México: un ejemplo de estructura agroganadera colonial, $1568-1810$ (Fronteras de la Historia, Vol. 15, Núm. 1, 2010), 61-84.

31 Valentina Garza Martínez y Juan Manuel Pérez Zevallos, El Real de Minas de San Gregorio de Mazapil, 1568-1700, (México, Instituto Zacatecano de Cultura Ramón López Velarde-Ayuntamiento de Mazapil 2005), 18.
} 


\section{FRONTERA DE GUERRA}

El poblamiento español significó el desplazamiento o asentamiento de los guachichiles, grupo nómada, que según las crónicas de la época eran los que habitaban en ese territorio; surgió una tensión territorial, manifestada en enfrentamientos. Sgún Powell, Mazapil, a poco tiempo de su descubrimiento se encontraba en peligro por los ataques de indios, esto ponía al lugar en una situación precaria. ${ }^{32}$

Los documentos de los siglos XVI y XVII representaron a los guachichiles como, belicosos y bárbaros. Por ejemplo, Pedro de Ahumada, describió a los guachichiles de Mazapil de la siguiente manera: "los primeros son los indios que llaman del Mazapil que están casi a la parte del nordeste de las minas de Zacatecas a veinte leguas de ellas [...] todos estos eran salteadores y de los que estaban concertados en la liga con los pueblos comarcanos de indios zacatecas que están cercanos a los pueblos de paz". ${ }^{33}$ Ante esta situación los españoles idearon un sistema para controlar a los guachichiles que les ocasionaban tantos problemas. Como parte de él se identificó a Mazapil, como una frontera, es decir un territorio que necesitaba de un programa de pacificación, instalando el presidio en 1573, y con ello, la consecuente presencia de capitanes y de protectores de indios; cuya función era la de pacificar a los indios, y de procurarles los elementos necesarios para su bienestar material y espiritual. ${ }^{34}$

La misión y los presidios, fueron las instituciones españolas más importantes en el territorio de frontera, ya que eran las encargadas de procurar la paz. El presidio, se instauró en la Nueva Galicia dos décadas después del inicio de la Guerra Chichimeca (1550-1590/92), fueron llamados al principio casas fuertes. Para 1569 el virrey Enríquez declaró que ya estaban en construcción las primeras, en Portezuelo y Ojuelos. ${ }^{35}$ Durante la década de los setenta se construyeron la mayoría de los presidios, que

\footnotetext{
32 Philip W. Powell, La Guerra Chichimeca (1550-1600), (México, Fondo de Cultura Económica 1997), 112.

${ }^{33}$ Román Gutiérrez, Sociedad y evangelización, 365.

34 Los estudios más recientes han permitido analizar los traslados de los guachichiles entre los diferentes lugares ya poblados por los españoles. Véase Lucas Martínez Sánchez, Guachichiles y franciscanos el libro más antiguo del convento de Charcas 1586-1663, (Saltillo, Coahuila: Gobierno del estado de Coahuila de Zaragoza/Secretaría de Cultura/Consejo Editorial del estado, 2019).

35 Philip Wayne Powell, "Génesis del presidio como institución fronteriza, 1569-1600" Estudios de Historia Novohispana (México:UNAM, No. 9, 1987), 21.
} 
podían ser fuertes amurallados o no fortificados, una de las funciones era la protección de los viajeros, que sufrían los ataques de los chichimecas.

En 1582, estancieros y capitanes hicieron un escrito, conocido como "la petición de los estancieros", en el que se solicitaba mayor protección, ya que los presidios no estaban cumpliendo con sus funciones, para demostrarlo le hicieron tres observaciones:

1. Los presidios eran a la sazón una aceptable y básica parte de la defensa, pero la protección de los caminos no era su única función. Algunos habían sido construidos (y otros recomendados) para defensa de los pueblos de los indios pacíficos (Jalapa y Xichú) y para protección de ganados (el del valle de San Francisco).

2. El presidio recomendado para el valle de San Francisco, que pronto se construyó, fue parcialmente cambiado desde su aspecto defensivo hasta un aspecto agresivo en la penetración a propósito a las tierras hasta ahora reconocidas como preserva de los guachichiles, la más fuerte y la más grande de las naciones chichimecas. Además de su papel de proteger ganado y vaqueros contra sus asaltos, se creía que tal proximidad a las rancherías guachichiles aminoraría sus ataques.

3. Aunque el valor de los presidios era reconocido ampliamente, se daba testimonio poco lisonjero acerca de la conducta de los soldados. ${ }^{36}$

Según esta declaración los presidios no estaban cumpliendo correctamente con su objetivo, de procurar y difundir la paz, que era función de los capitanes de paz, encargados de proteger a los indios chichimecas pacificados, lo que significaba cubrir sus necesidades físicas y espirituales, propagando el mensaje evangélico. Se tenía la idea de que no se levantarían si se les repartía las mercancías de paz: vestido, maíz, carne, sal, trigo, fríjol y herramientas para labrar la tierra. También les correspondía almacenar esos productos, mientras se repartían. Según Philip Powell los presidios del siglo XVI fueron:

Acaponeta (1581 o 1582), Aguascalientes (1575), F (fuerte) Atotonilco (1583), F. Bocas (1570-1571), F. Bocas de Maticoya (1590), F. Celaya (1570s), Chalchihuites (1581), Charcas (1581), Ciénega Grande (1570-1571), F. Cieneguilla de Portugal (1581), F (?) El Colegio (1583), F. Colotlán (1580s), F. Cuicillo (1570-1571), F. Fresnillo (1570s), Guanajuato (1570s), Jalpa (1576), F. Jaso Potrero de (1579), F. Jofre (15791580), F. Juchipila (1580s), Lagos (1581), Maguaos (1576-1577), F (?) Malpaso (15801581), Maxcala (1576), F. Mazapil(1570s), Nieto Paso de (1571-1572), Ojuelos (1570), F. Orirapúndaro (1580s), Palmar de Vega (1582), F. Palmillas (1570-1571), F.

36 Powell, Génesis del presidio, 29. 
Orirapúndaro (1580s), Tamaolipa (1599-1600), F (?) Tamaos (1576-1577), F. Tazazaca (1575), F (?) Felipe (1570s), San Francisco (región de Zimapán) (1580s), San Francisco Valle de (1582-1583), F. San Juan del Río (1582-1583), San Lorenzo de los Reyes (1570s), F. San Luis de la Paz (1590s), San Luis Potosí (1590s), San Martín (1580s), Santa Catarina Río de (1570s o 1580-1581), F, Santa María del Río (?) (1589- 1590), Sinaloa (1594-1595), F. Sombrerete (1580-1581), Tamaolipa (15901600), F. (?) Tamaos (1576- 1577), F. Tazazalca (1575), F. (?) Tecozautla, F. Tepezala (1573), F. Tlatenango (1581), Tolimán San Pedro de (1583-1585), Valles (1576-1577), Las Vueltas (1576), F. Xamay (1570s), F. Xichú (1583- 1584), Zimapán (1583-1584). ${ }^{37}$

Como parte de la comprobación de méritos que presentaban los capitanes, o en sus cuentas de guerra, se preguntaba a los testigos si habían cumplido con las funciones relativas con la pacificación. Ejemplo de ello son, las cuentas del capitán de Lizaldi, protector de indios en Mazapil, cuyo cargo tuvo en la década de los noventa del siglo XVI; declaró lo qué hizo con los cargos que se le entregaron: Martín de Lizaldi persona a cuyo cargo estuvo el almacén real de estas minas del Mazapil digo que en las cuentas que se me han tomado del ganado que distribuí a los indios del dicho almacén se me hizo cargo de los cueros y sebo del dicho ganado a que ofrecí dar información no ser a mi cargo y porque el ganado no tenía sebo y los cueros se habían llevado los indios". ${ }^{38}$

Estuvo a cargo del almacén real de Mazapil, donde se encontraban algunos bastimentos, como los cueros y el sebo, indispensables para las actividades en las minas. Una de las preguntas básicas era, ¿qué hacían los protectores de los indios, por proteger a los indígenas?, ya que como el título de su cargo lo indicaba tenían que cuidar la fe de los indios. Por lo que el testigo fue preguntado, "tenía obligación y si en esto ha tenido descuido en mirar por la conversión de ellos o no y si ha visto que en él haya habido falta o faltas y descuido de lo uno y de lo otro y no acudir cristianamente y como debe y están obligados y le está encargado y deben como leales vasallos y criados de su majestad". 39

Contestó que, "siempre ha visto este testigo acudir al dicho Martín de Lizaldi con solicitud y cuidado a las cosas tocantes con los dichos indios". ${ }^{40}$ En este caso la

\footnotetext{
${ }^{37}$ Powell, Génesis del presidio, 28.

${ }^{38}$ Archivo General de Indias (en adelante AGI), CONTADURÍA 851, Cuentas del capitán Martín de Lizaldi, protector de los indios de las minas del Mazapil en la frontera de los Chichimecas, 7.

${ }^{39} \mathrm{AGI}$, CONTADURÍA 851, 15:

${ }^{40} \mathrm{AGI}$, CONTADURÍA 851, 15:
} 
respuesta fue a favor del capitán. Las declaraciones hechas por distintos testigos, que están vertidas en las cuentas de guerra, permite observar a un capitán preocupado por los indios belicosos y por cuidar a los indios pacificados. En los libros de las cuentas de guerra de las tres últimas décadas del siglo XVI y las dos primeras del siglo XVII, se observa la presencia de la figura del capitán general y protector de indios, lo que permite identificar a Mazapil como un territorio de frontera de guerra.

En la década de los noventa del siglo XVI, Rodrigo Río de Loza, teniente de capitán general del reino de la Nueva Galicia, nombró capitán a Juan Morlete, sin importar que Francisco de Urdiñola fuera capitán en ese momento; quizá se debió al aumento de la necesidad de pacificar la frontera.

La presencia de dos capitanes indica también, el crecimiento del territorio de Mazapil, y también el aumento de los problemas causados por los indios salteadores. Rodrigo Río de la Losa, aclaró, que no era porque Francisco de Urdiñola haya hecho mal su trabajo, pero era necesario otro capitán, "Francisco de Urdiñola la de muchos a esta parte a servidos y está sirviendo a su majestad en las minas y comarcas del Mazapil, así en la guarda de aquellos vecinos de los que verifican las minas como de los caminos y personas que por ellos van y vienen y por la paz que ha asentado en los indios salteadores que él y vos habéis traído de paz a aquellas minas al presente hay tan solamente ocho soldados de presidio en la dichas minas". ${ }^{41}$

Se puede observar en este relato que el cargo de capitán, como todos los de esa época eran justificados por el servicio que significaban para el rey. Otro elemento de interés que encontramos es que la función benéfica que proporcionaba esa figura era para los vecinos y para los indios de paz. Continuando con la explicación del nombramiento de Morlete, de la Loza explicó que debido a que Urdiñola tenía que salir de Mazapil, era preciso alguien que estuviera allí cumpliendo las funciones de protector de indios.

Francisco de Urdiñola hace ausencia de las dichas minas ocupándose fuera de ellas en otras cosas del servicio de su majestad y estando él ausente conviene que haya persona que capitanes y rija y gobierne los dichos soldados del Mazapil y les mando lo que han de hacer y él los gobierne atento a lo cual por la presente

${ }^{41} \mathrm{AGI}$, CONTADURÍA 851, 18v-19. 
os doy comisión para que en cualquier ausencia o ausencias que hiciere el dicho capitán de las dichas minas del Mazapil vos como tal capitán de su majestad acudáis en todo punto de todas las cosas que el dicho capitán Francisco de Urdiñola acude están presente y vos por su ausencia rijáis y gobernéis los dichos soldados como de las manera que él lo hace con su presencia para el cual efecto mandó a el dicho capitán que cuando hubiere de hacer ausencia de las dichas minas. $^{42}$

Francisco de Urdiñola, desarrolló su labor de capitán en la frontera de la Nueva Galicia con la Nueva Vizcaya, y llegó a ser gobernador de este reino. Su movilidad resulta interesante, ya que su territorialidad no se centró en los límites administrativos. Llegó a ser uno de los hombres más ricos de la Nueva España, su riqueza perteneció al marquesado de Aguayo uno de los más importantes del siglo XVII.

Urdiñola nació en 1552, en el valle de Oyarzun, la provincia de Guipúzcoa, una de las vascongadas. Sus padres fueron Juanes de Urdiñola e Isabel de Larrmbide, Echenagucia Y Ugarte; sus abuelos paternos, Pedro Martín de Urdiñola Y María Juana de Baladarena y sus abuelos maternos, Juanes de Larrumbide y María Martín de Echenagucia. ${ }^{43}$

Algunas de las empresas exploratorias se llevaban a cabo con la inversión del capital de los capitanes, lo cual fungió como una estrategia de dominación y prestigio, por eso en esa carta Urdiñola declara haber invertido de su propia hacienda para la empresa del rey. El 23 de mayo de 1603, recibió el título de gobernador de la Nueva Vizcaya.

Otra estrategia fue llevar indios de paz a Mazapil, con el objetivo de contrarrestar los ataques de los guachichiles, y para tener mano de obra indígena. En una carta de 1576, el licenciado Rodrigo Río de la Loza, oidor y visitador de la audiencia de Guadalajara, refirió que los indios de paz en Mazapil, eran muchos, "los naturales del Mazapil, que son muchos y están en paz, y vendrían en conocimiento de nuestra fe católica”. ${ }^{44}$ En una cédula de 1591, se nombraron el gobernador, el alcalde y el alguacil

\footnotetext{
${ }^{42}$ AGI, CONTADURÍA 851, 21.

43 Vito Alessio Robles, Francisco de Urdiñola y el norte de la Nueva España, (México: Porrúa, 1981), 1.s[ẹp?

44 Luis Pedro Cantú, La provincia de los llanos, Charcas 1550 a 1610 (San Luis Potosí: COPOCYT/CONACULTA, 2005), 162.
} 
de los indios mexicas que vinieron de paz y que se establecieron en San Joseph de la Frontera.

\begin{abstract}
En México a treinta y uno de septiembre de mil quinientos noventa y un años se nombró por gobernador de los indios mexicanos que se van a poblar entre los chichimecas que vienen de paz y se asientan en el pueblo de San Joseph que es frontera de las minas de Mazapil don Simon Bernardo por tiempo de un año que consta desde el día de la data.

$\mathrm{Al}$ igual se nombraron el alcalde mayor, alguacil y el regidor. Nombramiento de alcalde para los mexicanos de la población de San Joseph (al margen) el dicho día se dio nombramiento de alcalde para los dichos indios mexicanos de la dicha población de San Joseph, al nombre Diego Mexicano como por se un año y tomó siguiente.

Nombramiento para regidor de los dichos indios (al margen) el dicho día se nombró por regidor para los dichos indios mexicanos de la dicha población a don Gabriel Jacobo indio mexicano. Por otro año. Nombramiento de alguacil mayor para los dichos indios (al margen) en el dicho día se dio nombramiento de alguacil mayor de los dichos indios mexicanos de dicha población de San Joseph a Melchor Suárez, indio. ${ }^{45}$
\end{abstract}

La idea de nombrar a indios con esos cargos, fue una para afianzar la pacificación en este territorio de frontera. Otro indicio de pacificación que se encuentra, es el hecho de que estos indios mexicas se poblaron en un pueblo llamado San Joseph de la Frontera, el cual no se ha podido relocalizar, ya que el documento señalado no lo ubica geográficamente, según el archivo histórico de localidades del Instituto Nacional de Estadística y Geografía, en el municipio de Mazapil actualmente sólo hay una comunidad que lleva el nombre de San José, nos referimos a San José de Carbonerillas, pero dudamos se refiera al de la Frontera.

En el Real de Minas de San Gregorio de Mazapil como asentamiento español se trasladaron las instituciones hispánicas: el presidio, el curato, la alcaldía. Durante las primeras décadas de vida de este Real, la relación con el espacio estuvo marcada por la tensión provocada por los dos grupos ocupantes, los guachichiles y los españoles; quizá aminorada con la presencia de indios de paz.

Se recordará que antes de la llegada de los españoles; Mazapil estaba poblado por guachichiles; Guillermo de las Casas los describió de la siguiente manera:

\footnotetext{
${ }^{45}$ Archivo General de la Nación (en adelante AGN), Instituciones coloniales, Indios, se nombra gobernador de los indios mexicanos que se van a poblar entre los chichimecas y se asienta en San José que es frontera de los indios del Mazapil, a don Simon Bernardo, por un año, Zacatecas. Pbo. San José (Vol. 5, Exp. 1005, 1591), 328v.
} 
Los guachichiles son luego inmediata, comienzan por la parte de Michoacán del Río grande y salen a yo (sic) el chico y valle de $S^{\circ}$ y los Arandas y sierras de las minas de Comanja y V(illa) de los Lagos, que es el nuevo Reyno de Jalisco, y toman las sierra de Xale y Bernal y tunal grande, por el límite de los guamares y bocas de Maticoya, las Salinas y Peñol blanco y Mazapil, y por las Macolias llegan hasta los confines de Panu(co)... (ocu)pan mucha tierra, y ansi es la mas gente de todos los chichimecas y que mas daños an hecho. Este nombre guachichil es puesto por los mexicanos, componose de -cabeza y colorado...ienen los cabellos con ello y porque de ellos usan (tr)aer unos bonetillos agudos de cuero colorado...iones de las jaulas que tienen las cabezas coloradas. Llaman guachichil. Ai entre estos guachichiles muchas parcialidades que por ser tierra tan larga no están todas bien sabidas. La más normada a sido la de Maticoya por aver andado Martinillo en ella con su gente y haber hecho tanto daño. También había otro gran Señor de mucha gente entre ellos, que se llamo Xale, este poseía lo mas del tunal grande, al cual sucedió barholomillo. También el Macolia es bien conocido y tratado de españoles, tiene mucha gente y tierra, este vivió en S. Francisco con Bartholomillo, y a entrambos los ahorco el Dr. Sande y en los Macolias sucedió un hijo suyo que cambien dice Macolia, y en la gente de barthomolillo. Anton Rayado. Hay otro que se dice Machiab con harta gente. Otro Guazcalo, Otro Maquimahal todos con gente de tequitatos, apartados y divisos por si y estos postreros tocan acá hacia los de Panuco, entre los Samues y Macolias, ai otros como guainame y los de las Salinas y Peñol Blanco que aun los españoles no tienen entera noticia, mas se hallan muchas rancherías divisas por el distrito (sic) de esta tierra y corre hasta la altura de 24 grados [...]Con estos chichimecas no se puede pelear con guerra descubierta, por andar siempre como andan huyendo escondiéndose en sierras, a rcabucos y quebradas y para poderlos prender siempre se procura hacerlo con engaños una veces tomándolos desapersividos y descuidados otras veces llamándolos de paz o viniendo ellos a tratar de ella, y dándoles palabra los engañan, prendiéndoles y cautivándoles.

[...] Poblarlos en tierra llana doctrinarlos en la ley de Dios y buenas costumbres dándoles todos los medios posibles para que consiga este fin que algunos de ellos son proveerlos de las cosas necesarias al sustento de la vida humana, pues de comer y vestir, y esto hasta que lo sepan hacer, y bastaría por solo un año, porque obligar a un bárbaro que viva en un páramo llano que en si ninguna cosa tiene de que sustentarse es obligarle a lo imposible porque de fuerza a de buscar de ciner pues el hambre le compele a ello y tomarlo donde lo hallare, pues verse desnudo entre vestidos tiene vergüenza y así huirán de nuestra conversación. ${ }^{46}$

Martín Despes, primer vicario de Mazapil, siguiendo los planteamientos de la época, encontró dos formas para pacificar a los guachichiles: la evangelización y la guerra, "aquellos indómitos salvajes se conviertan y vengan en conocimiento de la verdad en negocio importante que en Mazapil haya muchos cristianos"; 47 "el remedio para esto y las crueldades y muertes que por momentos hallen y libera bien que se les

\footnotetext{
46 González Dávila, editor, Guerra de los chichimecas, 12-26.

${ }^{47} \mathrm{AGI}$, Indiferente General $1092,1576-1577,2 \mathrm{~V}$.
} 
haga guerra de hecho y se den por esclavos perpetuos". ${ }^{48}$ Como se puede observar esta descripción de los guachichiles, habla de que era en términos conflictivos; también podemos inferir que se trata de los indicios de esa nueva manera de ocupación espacial.

\section{REFLEXIONES FiNALES}

La intención de estudiar a Mazapil como estudio de caso fue vislumbrar lo que ocurrió en la Nueva Galicia en relación a la conformación del territorio y la conformación de la frontera social, administrativa, geográfica y ambiental.

Después de lo analizado podemos decir que la transformación del paisaje en la Nueva Galicia durante el siglo XVI y XVII se dio bajo un proceso de conflictividad ambiental, reflejado en un conflicto bélico, denominado la Guerra Chichimeca, el que la historiografía lo ha identificado como político, pero que también fue social, territorial y ambiental. La conflictividad ambiental en Mazapil se representó en la defensa que hicieron los guachichiles por proteger su territorio, en el que construyeron su manera particular de relacionarse con el medio ambiente.

La transformación del paisaje en Mazapil no fue un proceso armonioso, sino que fue violento. La construcción territorial de la Nueva Galicia, fue un proceso progresivo, le dio otro rostro al paisaje. La idea de una jurisdicción administrativa, tanto civil como eclesiástica, como lo fueron la audiencia y el obispado, hicieron que sus habitantes conformaran una territorialidad particular

Pensemos que los españoles que llegaron a Nueva Galicia, habían pasado por la Nueva España, las maneras de relacionarse con su medio ambiente, se vieron modificadas en tierras novohispanas; al llegar a la Nueva Galicia se enfrentaron a un medio ambiente distinto, al cual por cuestiones de sobrevivencia debieron adaptarse y adaptar sus estrategias de conquista. En cuanto a los indígenas que habitaban el

${ }^{48} \mathrm{AGI}$, Indiferente General 1092 ,1576-1577, 3. 
territorio, a la llegada de los españoles vieron alterada su manera de relacionarse con el medio ambiente, las formas más intimas de su vida cotidiana se vieron violentadas; sufrieron cambios abruptos, pero no del todo aceptados, lo cual lo manifestaron de una forma belicosa. Este proceso lleno de rupturas decidí denominarlo conflictividad ambiental, caracterizado por el enfrentamiento de dos grupos sociales completamente distintos.

Fue un juego de alteridades, dentro del cual se fueron rolando el papel del otro, el extraño. Para los españoles no fue nada sencillo enfrentarse ante lo desconocido, cada lugar descubierto significaba un hallazgo, en el sentido estricto de la palabra, era el ir construyendo un territorio, que se imaginaba aún estaba por encontrarse; se tenía la idea de que aún había Tierra Adentro. El asombro de los indígenas se debió al ir viendo la transformación del paisaje, la construcción de casas, el transporte sobre animales, que no conocían, y aún más el comérselos, según las crónicas de la época, esto sucedió rápidamente, incluso los asaltos a las diligencias españolas, para hurtar al ganado fue otro elemento en el proceso denominado conflictividad ambiental. Pero la adaptación de los indígenas a esas maneras ambientales y territoriales, no fue rápida, sino que pasaron por un proceso de resistencia, de defensa.

Según los datos de la visita de 1608 a 1610, del oidor Gaspar de la Fuente, encontró que ya había algunos guachichiles poblados en Cedros, por lo cual ya eran parte del poblamiento español. Los guachichiles eran el grupo indígena que predominaba en el territorio de Mazapil, o por lo menos su presencia fue muy mencionada en los documentos de la época. Encontramos la existencia de otras comunidades, como los Zabanos que habitaban en el Pico del Teyra; la información acerca de ellos es escasa. Según los datos proporcionados por Martín Despes, en su crónica, para la década de los setenta del siglo XVI, ya habían sido pacificados.

En Mazapil por su ubicación de frontera, fue necesario instituir un presidio en 1573, debido al conflicto con los indios de guerra, según las noticias de la época, en su mayoría eran guachichiles. Una de las estrategias para controlarlos fue llevar indios de paz, a los que era necesario otorgarles algunos artículos para la vida cotidiana, como: maíz, ropa, fríjol y carne. Los capitanes de guerra encargados de hacer ese reparto, 
tenían la obligación de entregar una relación de lo otorgado. Este proceso fue analizado con un enfoque microhistórico, pero concibiéndolo como parte del entramado macrohistórico, es decir esta situación privó en la Nueva Galicia; dónde había tierras por explorar e indios por pacificar; la conflictividad ambiental fue parte de la cotidianeidad de sus habitantes durante el siglo XVI y las primeras décadas del XVII.

Mazapil fue la raya de la Nueva Galicia, término utilizado en la época virreinal para definir a la frontera administrativa, pero como hemos analizado también fue social, geográfica y ambiental; fue una raya movible y permeable.

Quiero terminar este artículo, dedicándolo a, Juan Manuel Pérez Zevallos, quien murió hace unos días. Sus trabajos fueron pioneros en la historiografía de Mazapil; gracias a sus recopilaciones de documentos es que podemos contar con parte fundamental de la memoria histórica del Real de Minas de San Gregorio de Mazapil.

\section{REFERENCIAS}

Alessio Robles, Vito, Francisco de Urdiñola y el norte de la Nueva España, (México: Porrúa, 1981).

Buenrostro Ceballos, A. F, ed., Memoria del I Congreso Internacional sobre Fronteras en Iberoamérica: Ayer y hoy (Tijuana: Universidad Autónoma de Baja California, Tomo 1).

Cantú, Luis Pedro, La provincia de los llanos, Charcas 1550 a 1610 (San Luis Potosí: COPOCYT/CONACULTA, 2005).

Cramaussel, Chantal, Poblar la frontera. La provincia de Santa Bárbara en Nueva Vizcaya durante los siglos XVI y XVII, (México, El Colegio de Michoacán: 2006).

Diccionario de la lengua castellana, en que se explica el verdadero sentido de las voces, su naturaleza y calidad, con las frases o modos de hablar, los proverbios o refranes, y otras convenientes al uso de la lengua, dedicado al Rey Nuestro Señor Felipe V. (que Dios lo guarde), (Madrid: Real Academia Española por los Herederos de Francisco del Hierro, 1737. Edición Facsimilar, ed. Gredos, 1990, T. 1).

Folchi D, "Conflictos de contenido ambiental y ecologismo de los pobres: no siempre pobres, ni siempre ecologistas" Ecología Política (22 2001), 79-101. 
Fonseca Hernández, Raúl, Wilson Picado Umaña, Abelardo Morales Gamboa y Maximiliano López López, eds., Cruce de caminos. Lecturas disciplinarias del territorio (San José de Costa Rica: FLACSO Costa Rica/Universidad Nacional de Costa Rica: 2020).

Garza Martínez Valentina y Juan Manuel Pérez Zevallos. El Real y Minas de San Gregorio de Mazapil 1568-1700 (Zacatecas, México: Instituto Zacatecano de Cultura Ramón López Velarde-Ayuntamiento de Mazapil: 2004).

González Dávila, Gil, editor, Guerra de los chichimecas (México: Gobierno del Estado de Jalisco/Universidad de Guadalajara/Instituto Jalisciense de Antropología e Historia 1994).

Hernández Madrid Miguel J. y José Lameiras Olvera (eds.), Las ciencias sociales y humanas en México. Síntesis y perspectivas del fin de siglo (México: El Colegio de México, 2000).

Los Municipios de Zacatecas. Memoria y patrimonio (Zacatecas, México: Crónica del estado de Zacatecas, 2019).

Martínez Sánchez, Lucas, Guachichiles y franciscanos el libro más antiguo del convento de Charcas 1586-1663, (Saltillo, Coahuila: Gobierno del estado de Coahuila de Zaragoza/Secretaría de Cultura/Consejo Editorial del estado, 2019).

Powell, Philip Wayne, "Génesis del presidio como institución fronteriza, 1569-1600" Estudios de Historia Novohispana (México:UNAM, No. 9, 1987).

Powell, Philip Wayne, La Guerra Chichimeca (1550-1600), (México, Fondo de Cultura Económica 1997).

Rangel Silva, José Alfredo, "Capitanes a guerra, linajes de frontera. Estrategias de dominación entre las élites familiares en el oriente de San Luis, 1617-1823", México, (Tesis doctoral, El colegio de México, 2006).

Román Gutiérrez, José Francisco, Sociedad y evangelización en la Nueva Galicia durante el siglo XVI (Guadalajara: Instituto Nacional de Antropología e Historia/El Colegio de Jalisco/Universidad Autónoma de Zacatecas, 1993).

Panico Francesco y Caludio Garibay Orozco, "Mazapil, Zacatecas, México: un ejemplo des estructura agroganadera colonial (1568-1810)", Fronteras de la Historia 15 (Número $12010), 61-84$.

Salas Hernández, Juana Elizabeth y Margil de Jesús Canizales Romo, Coords., Historia Ambiental en el Norte de México (México, Universidad Autónoma de Zacatecas, El Colegio de San Luis A.C. y La Red Columnaria, 2020).

Sheridan Prieto, Cecilia, "Reflexiones en torno a las identidades nativas en el noreste colonial", Relaciones 92, (Otoño 2002, Vol. XXIII), 76-106. 
Solano, Francisco de y Salvador Bernabéu coords., Estudios (nuevos y viejos sobre la frontera) (Madrid: Consejo superior de investigaciones científicas-centro de estudios históricos, departamento de América 1991).

Taracena Arríola, Arturo, "Región e historia", Desacatos. Revista de antropología social, revista semestral, (primavera de 1999), 28-31.

\title{
Real de Minas de San Gregorio de Mazapil, border of the Kingdom of Nueva Galicia
}

\begin{abstract}
The territorial conformation of the Hispanic Monarchy was constituted by a process of territoriality and deterritoriality, processes that were marked by the conformation of administrative, social, imaginary, geographical and environmental borders, mutable in space and time. The Kingdom of Nueva Galicia in its territorial conformation during the 16th-17th centuries was in constant expansion and conquest and therefore the Land Within was modified, and with it the border. San Gregorio de Mazapil was a Royal of Mines, which since its foundation in 1568, served as an administrative border between the Kingdom of Nueva Galicia and that of Nueva Vizcaya, but it was also a social, war, geographical and environmental border. The article analyzes this Real de Minas as a case study, and reflects on these categories, concentrating them on the social, war, geographical and environmental frontier, to understand the settlement process and the transformation of the landscape during the 16th-17th centuries.
\end{abstract}

Keywords: territory; border; transformation of the landscape; Nueva Galicia.

Recibido: $11 / 11 / 2020$ Aprobado: 08/02/2021 